\title{
Data Discrimination in Fault-Prone Sensor Networks
}

\author{
Xiaoning Cui ${ }^{1,2,3,4,5}$, Qing $\mathrm{Li}^{4,5}$, Baohua Zhao ${ }^{1,2,3,4}$ \\ ${ }^{1}$ School of Computer Science and Technology, University of Science and Technology of China, Hefei, China \\ ${ }^{2}$ State Key Laboratory of Networking and Switching Technology, Beijing, China \\ ${ }^{3}$ Province Key Laboratory of Software in Computing and Communication, Hefei, China \\ ${ }^{4}$ Joint Research Lab of Excellence, CityU-USTC Advanced Research Institute, Suzhou, China \\ ${ }^{5}$ Department of Computer Science, City University of Hong Kong, Hong Kong, China \\ E-mail:cxning@mail.ustc.edu.cn,itqli@cityu.edu.hk,bhzhao@ustc.edu.cn \\ Received January 31, 2010; revised February 22, 2010; accepted February 24, 2010
}

\begin{abstract}
While sensor networks have been used in various applications because of the automatic sensing capability and ad-hoc organization of sensor nodes, the fault-prone characteristic of sensor networks has challenged the event detection and the anomaly detection which, to some extent, have neglected the importance of discriminating events and errors. Considering data uncertainty, in this article, we present the problem of data discrimination in fault-prone sensor networks, analyze the similarities and the differences between events and errors, and design a multi-level systematic discrimination framework. In each step, the framework filters erroneous data from the raw data and marks potential event samples for the next-step processing. The raw data set $D$ is finally partitioned into three subsets, $D_{\text {event }}, D_{\text {error }}$ and $D_{\text {ordinary }}$. Both the scenario-based simulations and the experiments on real-sensed data are carried out. The statistical results of various discrimination metrics demonstrate high distinction ratio as well as the robustness in different cases of the network.
\end{abstract}

Keywords: Data Discrimination, Fault-Prone Sensor Network, Event, Error, Distinction Ratio

\section{Introduction}

One of the major applications of sensor networks is event detection [1], while the data uncertainty caused by faulty sensors increases the difficulty of distinguishing between events and errors in sensor data, and correspondingly affects the design of data processing framework in a sensor network. Due to the rather limited resource and fault-prone characteristics in sensor networks, the design principle of sensor data processing mainly lies in simple operation, fault tolerance, distributed processing and efficient distinction between erroneous measurements and events $[2,3]$. The major techniques of anomaly detection include histogram-based method [4], kernel estimation $[5,6]$, ranking/score-based method $[7,8]$, dependency analysis [9], and etc. There are also some extensive research works on the region detection of anomalous sensor readings $[10,11]$. However, to the best of our knowledge, most of these works either separate event detection and error detection into two problems or ambiguously perceive events and errors as anomalies. According to the summary of the state-of-the-art anomaly detection techniques [12], there lack sufficient concern of the discrimination between events and errors in sensor data processing [3]. Therefore, in this article, we focus on designing a discrimination framework to solve this problem.

The rest of the article is organized as follows. In Section 2 , the problem is analyzed based on the similarities and differences between events and errors. In Section 3, a discrimination framework is illustrated. The performance of the framework is evaluated by two scenariobased simulations and a series of experiments on a real-world sensor dataset in Section 4, and finally, Section 5 concludes the article with some discussions on the potential extension of the framework.

\section{Problem Analysis}

The problem of distinguishing between events and errors has been investigated based on a few common assumptions [13]: 1) the network holds a hierarchical structure and sensor data are forwarded to nearby local fusion centers to handle data processing; 2) all of the data received by the fusion center are not corrupted by any communication fault; 3 ) there are no malicious attacks 
on the sensor network. The general discrimination problem can thus be defined via Definitions 1 and 2 .

Definition 1 Sensor Data Sample: A sensor data sample smp is a 5-tuple: < dtype, value, time, location, $s \mathrm{ID}>$, where dtype reflects the physical meaning of the data (e.g., temperature data and concentration data are of different dtype), value is the data value, time and location express the sampling condition, and $s$ ID is the sensor index.

Definition 2 Discrimination of Events and Errors (DEE): Given a set of sensor data $=\{s m p\}$ from a certain area $S$ during a certain period of time $T$, $\underline{\mathrm{DEE}}$ finds an event dataset $D_{\text {event }}$, an error dataset $D_{\text {error }}$ and an ordinary dataset $D_{\text {ordinary }}$, where $D=D_{\text {event }} \cup D_{\text {error }} \cup D_{\text {ordinary. }}$.

According to the classification of sensor data errors in [13], Table 1 lists the event types that are easily to be confused with the corresponding error types.

On the one hand, the corresponding types in Table 1 show the representational similarities of events and errors under certain conditions. On the other hand, due to the different causes of the occurrence of events and errors, an event does not occur frequently and usually changes historical pattern of sensor data, while an error may occur frequently in a sensor network and the erroneous data measurement is normally represented as an arbitrary change [3].

\section{Discrimination Framework}

In view of the similarities and differences of event and error readings, we present in this section a discrimination framework to solve the DEE problem from a data-mining perspective, with the following assumptions: 1) The sensor network is classified into clusters with a certain amount of sensor nodes in a cluster and one local fusion center in charge of one cluster; all of the fusion centers have path to the base station. 2) An event can be detected within at least one cluster; at least $m$ ( $m$ is an integer and $m>k / 2$ ) sensor nodes can detect the same event during the same time unit, where $k$ is the average number of

Table 1. Event and error types.

\begin{tabular}{|c|c|}
\hline Event & Error \\
\hline $\begin{array}{l}\text { Incidental event: } \\
\text { Occurs without any sign. } \\
\text { e.g., irregular heart-beat rate. }\end{array}$ & $\begin{array}{l}\text { Discrete error: } \\
\text { An isolated data sample that } \\
\text { significantly deviates from } \\
\text { other observations. }\end{array}$ \\
\hline $\begin{array}{l}\text { 1) Instantaneous event: } \\
\text { Occurs suddenly and lasts a } \\
\text { relatively short period of time. } \\
\text { e.g., car accident. } \\
\text { 2) Durative event: } \\
\text { Occurs gradually and lasts a } \\
\text { relatively long time. } \\
\text { e.g., fume diffusion. }\end{array}$ & $\begin{array}{l}\text { Continuous error: } \\
\text { 1) Spike: } \\
\text { A rate of change much greater } \\
\text { than expected over a short } \\
\text { period of time. } \\
\text { 2) Stuck: } \\
\text { A series of data with zero value } \\
\text { or almost zero variation for a } \\
\text { period of time greater than } \\
\text { expected }\end{array}$ \\
\hline
\end{tabular}

one-hop neighbors of one sensor node in the network. 3) The chance for $k$ neighboring sensor nodes to make the same type of errors simultaneously is represented by a very small positive number $\varepsilon$.

There are various ways to evaluate the anomalous data samples, e.g., histogram method, kernel estimation, or ranking analysis. In the histogram method [4], a series of value ranges are set before data processing. All of the data samples are put into the corresponding value range and a histogram is generated to record the occurrence frequency of each value range. If the occurrence frequency exceeds certain threshold, it is recognized as an event/error sample. In the kernel estimation [5,6], all of the data samples form a kernel to represent the major value distribution. The samples with the values far from the kernel value are recognized as event/error samples. In the rank-based methods $[7,8]$, the relations of two sensor data samples are described by links. The strength of a link is determined by the correlation of dtype and value of the samples. A sensor data sample is recognized as an event/error sample if it has weak link to other data samples generated from neighboring nodes. These methods either need global knowledge of all of the sample data for estimation or lack a systematic view of the network structure. In our work, we attempt to integrate the advantages of these approaches and avoid their disadvantages. On the basis of the assumptions mentioned above, the workflow of the discrimination framework is described in Figure 1.

The framework includes four steps, representing four levels of data-processing granularity: Node level, neighbor level, cluster level and network level. The key point is to utilize the occurrence frequency and the change of pattern to distinguish errors and events. Considering the computing capability and energy constraints of sensor nodes as well as the response requirement of the monitored events, different methods are suitable for different levels (Figure 1).

1) Node level: Temporal processing is conducted on a sequence of historical samples on a sensor node. If the value or gradient of a sample exceeds the range of some physical constant, it is apparently an erroneous sample

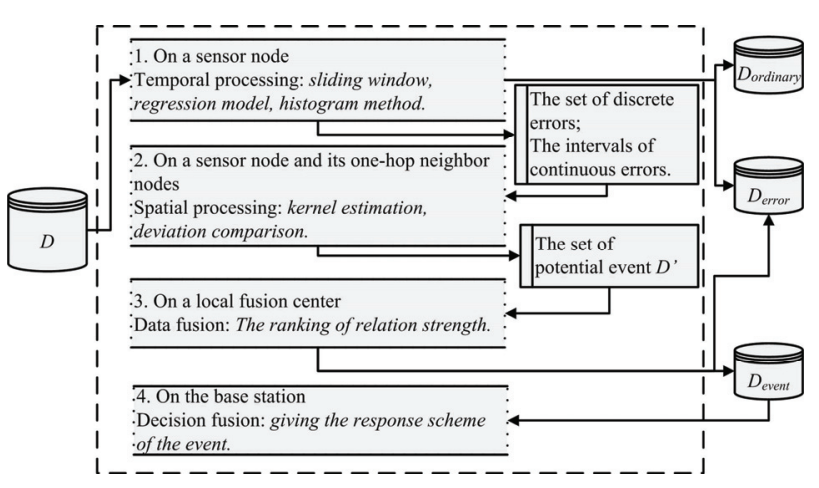

Figure 1. The workflow of the discrimination framework. 
that should be put into $D_{\text {error }}$. Otherwise, the set of discrete errors are picked out and the samples of continuous errors are marked by interval for further processing. In our work, linear regression is used based on a fixed size of sliding window. The value differences of the predicted samples and the real-sensed samples reflect the temporal pattern of the sample sequence. Both events and errors would incur significant change of pattern and thus a higher value difference in prediction, according to which the involved samples are marked for further discrimination. The rest of the samples are put into $D_{\text {ordinary }}$.

2) Neighbor level: Spatial processing is used to compare the samples with discrete errors or continuous errors over neighboring nodes. Based on the assumptions in this section, an anomalous sample probably reflects an event if there is enough number (the number is set to be equal to or over $50 \%$ in our simulation and experiment) of neighboring nodes reporting the same data exception. Such samples are put into the set of potential events $D$ '. Other anomalous samples belong to $D_{\text {error }}$.

3) Cluster level: The local fusion center evaluates the samples in $D^{\prime}$ with reference to $D_{\text {ordinary. }}$ The event samples are finally selected from $D^{\prime}$ and constitute $D_{\text {event }}$. In our work, we use deviation-based ranking strategy to evaluate the samples in $D^{\prime}$ because it has been assumed that there is little chance for all of the nearby nodes (within a cluster) to get similar wrong readings. The samples in $D \backslash D_{\text {event }}$ are added to $D_{\text {error }}$.

4) Network level: The base station gets $D_{\text {event }}$ and makes decision fusion [14], i.e., taking action according to the event information. Since it does not involve the DEE problem, this level is beyond the scope of this paper.

\section{Performance Evaluation}

The performance of the discrimination framework is evaluated by two scenario-based simulations in Subsection 4.1 and an experiment based on real-sensed data in Subsection 4.2 .

\subsection{Scenario-Based Data Simulations}

Scenario 1. Irregular heart-bit rate: The heart-bit rate depicts how many times one's heart beats per minute. A human being usually keeps a stable heart-bit rate with little fluctuations, represented as $f(t)=c \pm \varepsilon_{c}$, where $t$ is the moment when measuring the heart-bit rate, $c$, and $\varepsilon_{c}$ is a small positive number to represent fluctuations. Irregular heart-bit rate means that the fluctuation exceeds the normal range, $\left[-\varepsilon_{c}, \varepsilon_{c}\right]$. The simulation based on Scenario 1 aims at evaluating the performance on discriminating discrete errors with incidental events (Table 1). Considering the requirement of the report correctness and the response time, a small-scale network is simulated with less than 10 sensor nodes. Meanwhile, the node- level (Step 1) and neighbor-level (Step 2) processing are employed in the data discrimination.

In Table 2, the error occurrence rate is the ratio of the amount of erroneous samples over the total amount of the samples. It is set from $10 \%$ to $50 \%$, which is large enough compared with the event occurrence rate $(\leq 1 \%)$. This is consistent with the frequency difference of errors and events. A sample is recognized as either an ordinary sample or a potential-event sample in node-level processing, except those which exceed the normal value range. The neighbor level processing further discriminates events and errors in the potential-event sample set. Therefore, $c_{6}$ is the distinction ratio in node-level processing (Step 1) and the sum of $r_{7}, r_{8}$ and $r_{9}$ is the distinction ratio in neighborlevel processing (Step 2).

Given a set of sensor nodes measuring the heart-bit rate of a person, the parameters are configured following Table 2. Specifically, the simulations are conducted through the following two cases.

Case 1. Slower heart-bit rate

Figure 2 shows the comparison of the distinction

Table 2. Parameter configuration of Scenario 1.

\begin{tabular}{|c|c|}
\hline & Parameters \\
\hline $\begin{array}{c}\text { General } \\
\text { parameters }\end{array}$ & $\begin{array}{l}\text { Data amount: } 1.44 \times 10^{7} \text { heart-bit rate samples } \\
\text { per node with error/event injection; } \\
\text { Sampling frequency: } 1 \mathrm{kHz} ; \\
\text { Error occurrence rate } \leq 50 \% \text {; } \\
\text { Error type: discrete error; } \\
\text { Event occurrence rate } \leq 1 \% \text {; } \\
\text { Event type: incidental event; } \\
\text { Normal heart-bit rate value range: }[60,100] \text {; } \\
\text { Error/Event value range: [50,60) for slower } \\
\text { heart-bit rate and }(100,199] \text { for faster heart-bit } \\
\text { rate. }\end{array}$ \\
\hline $\begin{array}{c}\text { Step 1 } \\
\text { (node level) }\end{array}$ & $\begin{array}{l}\text { Window length: } 4000 \text { samples(data in } 4 \\
\text { seconds); } \\
\text { Sliding ratio: } 25 \% \text { (1000 samples, data in } 1 \\
\text { second). }\end{array}$ \\
\hline $\begin{array}{l}\text { Evaluation } \\
\text { metrics } \\
\text { (for Step1) }\end{array}$ & $\begin{array}{l}c_{1} \text {-mistaking errors for ordinary samples; } \\
c_{2} \text {-marking errors as potential events; } \\
c_{3} \text {-marking ordinary readings as potential } \\
\text { events; } \\
c_{4} \text {-mistaking events for ordinary samples; } \\
c_{5} \text {-marking events for potential events; } \\
c_{6} \text {-correct distinction of ordinary samples; } \\
c_{1}+c_{2}+c_{3}+c_{4}+c_{5}+c_{6}=1 \text {; } \\
\text { Distinction ratio (Step } 1)=c_{2} \text {. }\end{array}$ \\
\hline $\begin{array}{c}\text { Step } 2 \\
\text { (neighbor level) }\end{array}$ & Average number of 1-hop neighbors: 2 \\
\hline $\begin{array}{l}\text { Evaluation } \\
\text { metrics } \\
\text { (for Step2) }\end{array}$ & $\begin{array}{l}r_{1} \text {-mistaking errors for ordinary samples; } \\
r_{2} \text {-mistaking errors for events; } \\
r_{3} \text {-mistaking ordinary readings for events; } \\
r_{4} \text {-mistaking ordinary readings for errors; } \\
r_{5} \text {-mistaking events for ordinary samples; } \\
r_{6} \text {-mistaking events for errors; } \\
r_{7} \text {-correct distinction of error samples; } \\
r_{8} \text {-correct distinction of events samples; } \\
r_{9} \text {-correct distinction of ordinary samples; } \\
r_{1}+r_{2}+r_{3}+r_{4}+r_{5}+r_{6}+r_{7}+r_{8}+r_{9}=1 \text {; } \\
\text { Distinction ratio }\left(\text { Step } 2 \text { ) }=r_{7}+r_{8}+r_{9} \text {; }\right. \\
\text { False Alarm }=r_{2}+r_{3} \text {; Miss Hit }=r_{5}+r_{6} \text {. }\end{array}$ \\
\hline
\end{tabular}




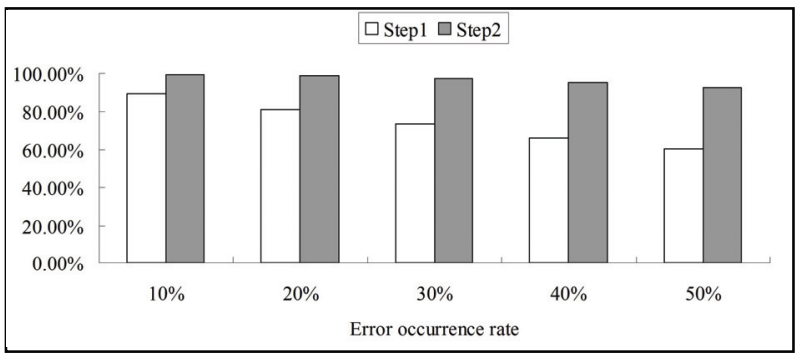

Figure 2. Distinction ratio in each step of Case 1.

ratios after Steps 1 and 2. The distinction ratio after Step 1 declines with the increase of error occurrence rate, from $89.78 \%$ (when error occurrence rate $=10 \%$ ) to $60.3 \%$ (when error occurrence rate $=50 \%$ ). However, Step 2 achieves similar distinction ratio over $90 \%$ even though the error occurrence rate is as high as $50 \%$.

Moreover, as shown in Figure 3, the distinction ratios of event and ordinary samples both approach $100 \%$. The distinction ratio of errors slightly goes down from $97 \%$ (when error occurrence rate $=10 \%$ ) to $84 \%$ (when error occurrence rate $=50 \%$ ).

Furthermore, Figure 4 shows the false-alarm rate and the miss-hit rate for Case 1. On one hand, the false-alarm rate grows larger with the increase of the error occurrence rate. The statistical result has shown that the increase of error occurrence rate has accelerated the growing of the false-alarm rate. On the other hand, there is almost no miss-hit in the data set.

Case 2. Faster heart-bit rate

Case 2 tests the performance on faster heart-beat rate monitored by sensor networks. It is similar to Case 1, except that the value ranges of event samples are different. Figure 5 shows the step-wise comparison of the distinction ratios. The trends are similar to that of Figure 2, i.e., the distinction ratio varies from $90.39 \%$ (when error occurrence rate $=10 \%$ ) down to $59.83 \%$ (when error occurrence rate $=50 \%$ ) after Step 1, and the ratio increases to at least $93.31 \%$ after Step 2. When error occurrence rate $=10 \%$, such a ratio achieves as high as $99.70 \%$.

Figure 6 compares the distinction ratio of error samples, event samples and ordinary samples in Case 2. Similarly to that in Case 1, the distinction ratio of errors declines with the increase of erroneous data amount in the data set, and the distinction of ordinary samples keeps a stable ratio of near $100 \%$. The event samples are well discriminated under most conditions except when error occurrence rate $=30 \%$, where $1 / 6$ event samples are mistaken for error samples. This is because, in the test case, many of the event samples are cross-occurred with error samples within the same sliding window. It means that the distinction ratio of event samples is not affected by the error occurrence rate, but by when and where an event and/or an error occur.

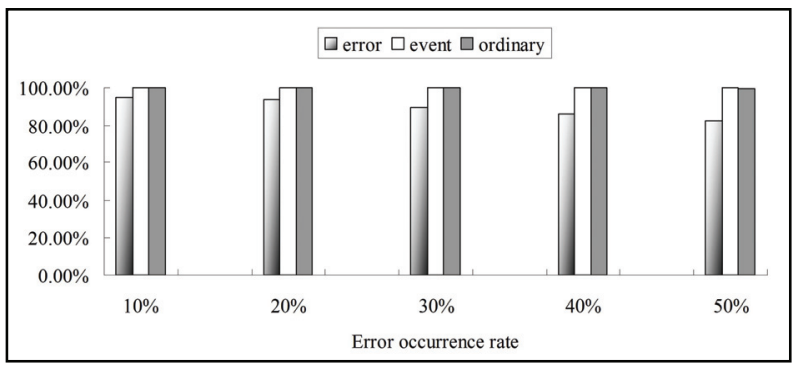

Figure 3. Comparison of distinction ratio of error $\left(r_{7} /\left(r_{1}+r_{2}\right.\right.$ $\left.\left.+r_{7}\right)\right)$, event $\left(r_{8} /\left(r_{5}+r_{6}+r_{8}\right)\right)$ and ordinary samples $\left(r_{9} /\left(r_{3}+r_{4}\right.\right.$ $\left.+r_{9}\right)$ ) of Case 1 .

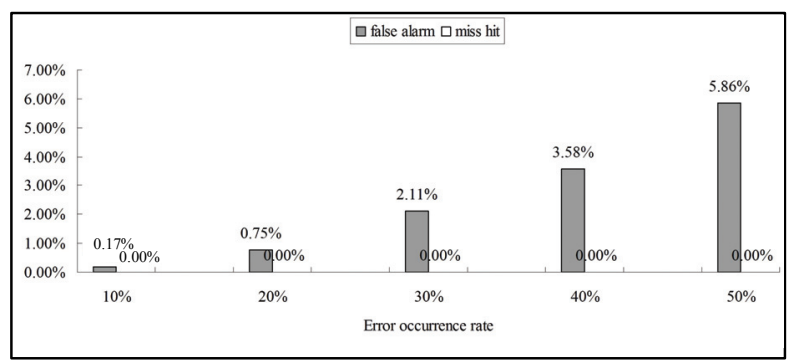

Figure 4. False-alarm and miss-hit rate of Case 1.

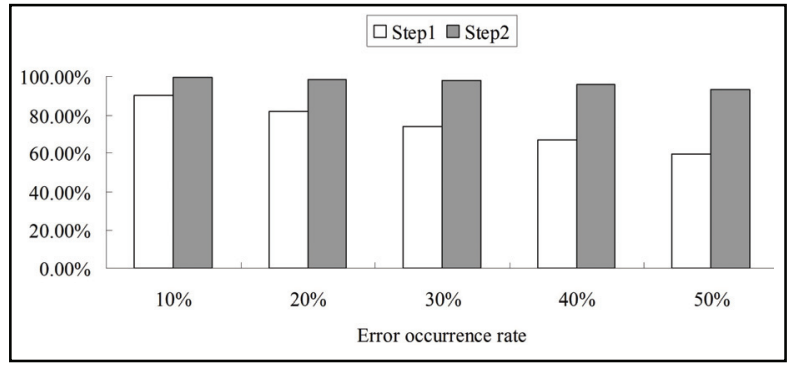

Figure 5. Distinction ratio in each step of Case 2.

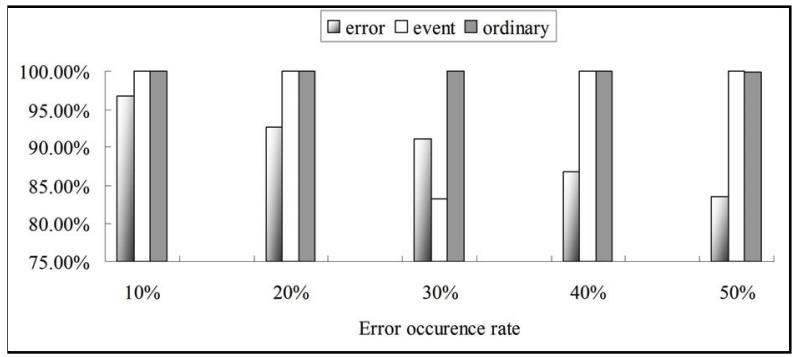

Figure 6. Comparison of distinction ratio of error, event and ordinary samples of Case 2.

Figure 7 further gives the false-alarm rate and misshit rate of Case 2. The values and trends are similar to that in Case 1.

Scenario 2. Car accident: In the industry of car manufacture, accidents occur often due to acceleration. Experience tells that, when the sampling rate $=1 \mathrm{kHz}$ and the absolute acceleration (In the rest of this paper, we use the word "acceleration" to represent "absolute accelera- 
tion" for short.) exceeds $47 \mathrm{~m} / \mathrm{s}^{2}$ between two consecutive samples, car collision is expected to happen (see, e.g., Figure 8 when time $=8 \mathrm{~ms}$, acceleration $=50 \mathrm{~m} / \mathrm{s}^{2}$ ). Meanwhile, the response time of a car accident should be less than $20 \mathrm{~ms}$ so that the air bag could be triggered and the driver could have enough time to take action.

There are usually 4 sensor nodes located at 4 wheels of a car and a fusion center to integrate the samples, so node-level (Step 1) and cluster-level (Step 2) processing are employed in the simulation. Considering the change pattern of the acceleration in Figure 8, an integration-based method is used in order to separate event samples from erroneous samples, i.e., calculate the integration of the sample values within a sliding window using Formula (1), and check whether the numerical integration exceeds a certain threshold:

$$
S(n, k)=\frac{1}{f} \sum_{i=n-k+1}^{n} a(i)
$$

where $f$ is the sampling rate, $n$ is the total amount of samples and $k$ is the window length. When $f=1 \mathrm{kHz}$ and $k$ is set to $7 \mathrm{~ms}$, the threshold is calculated by $0.5 \times 7 \times 47=$ 164.5 , indicating the linear changing process of the acceleration from $0 \mathrm{~m} / \mathrm{s}^{2}$ to $47 \mathrm{~m} / \mathrm{s}^{2}$ within a sliding window.

Similar to Table 2, Table 3 lists the parameter configuration of the simulation.

Figure 9 compares the distinction ratio of error samples, event samples and ordinary samples of Scenario 2. Since both the event pattern and the error types are more complex in Scenario 2 than that in Scenario 1, the distinction ratios are generally lower than those in Scenario 1. However, the trends are the same, i.e., higher-level processing will increase the distinction ratio.

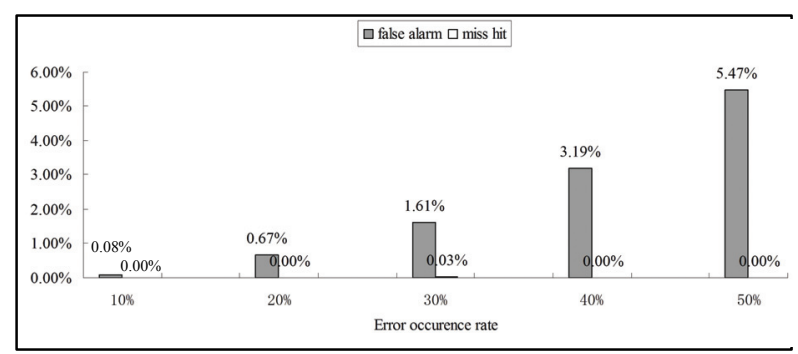

Figure 7. False-alarm and miss-hit rate of Case 2.

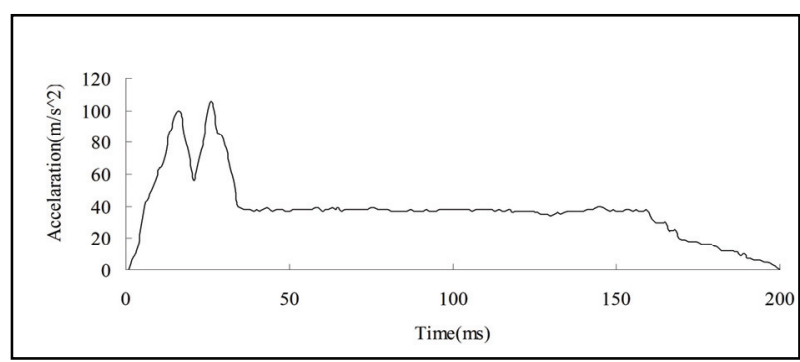

Figure 8. The change pattern of the acceleration in a car accident.
Table 3. Parameter configuration of Scenario 2.

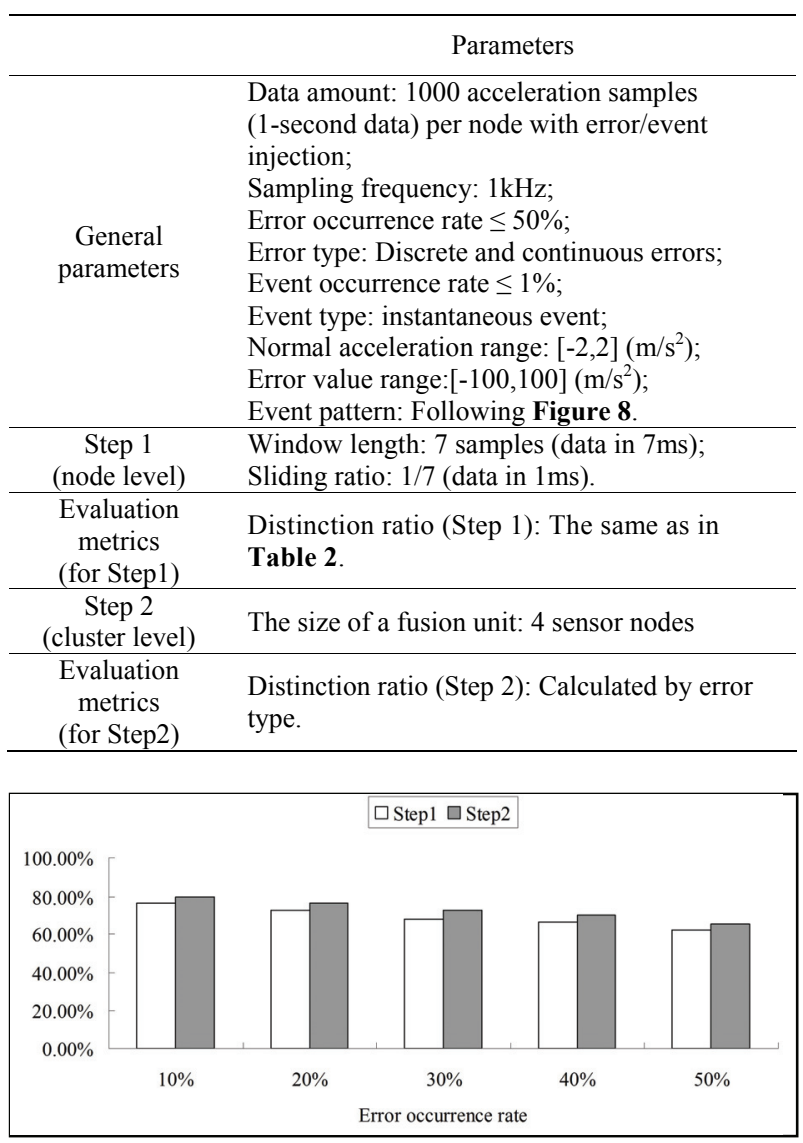

Figure 9. Distinction ratio in each step of Scenario 2.

Furthermore, Figure 10 compares the distinction ratios of discrete error (79.59\% in average) and continuous error ( $84.80 \%$ in average). It is shown that, the distinction ratio varies with different error occurrence rate in the network, but there is no significant sign to tell which type of errors could be better discriminated in the data with a complex event pattern. The performance of data discrimination largely depends on the relative occurrence time and location of errors and events. For example, in the test case, when a continuous error occurs next to the event samples, the processing tends to misjudge such erroneous samples. Meanwhile, if most of the neighboring nodes are generating error samples, it is difficult for the fusion center to report correct discrimination results.

\subsection{Real-Sensed Data Experiment}

Our experiment aims at testing the performance on the discrimination of durative event samples. The experiment is carried out based on the sensor data collected by the Intel Berkeley Research Lab [15], where we select the data generated from a subset of the sensor nodes and inject errors and events to the raw data to evaluate the 
performance of the distinction framework. The experiment includes three steps, corresponding to the processing of the node, neighbor and cluster levels, respectively. Without loss of generality, both the error probability of a sample and the event pattern are unknown to a sensor node. The parameters used in the experiment are listed in Table 4.

The discrimination metrics in Table $\mathbf{4}$ are tested for the sample prediction in Step 1 and the event discrimination of Steps 2 and 3. Figure 11 shows the accuracy comparison of these metrics according to the experiment on four nearby nodes (notes 1, 2, 3, and 33 in [15]) with about 2000 temperature samples per node. Due to the miss-sampling and over-sampling cases, the integrated raw data have 2787 samples. The discrimination metrics are calculated by sliding window. The correlation coefficient is shown as the best metric because of its stable performance over both little-changed samples (e.g., the samples during time intervals [2989, 3633], [4740, 5566]) and fast response over dynamically changed samples (e.g., the samples during time interval [3689, 3904]).

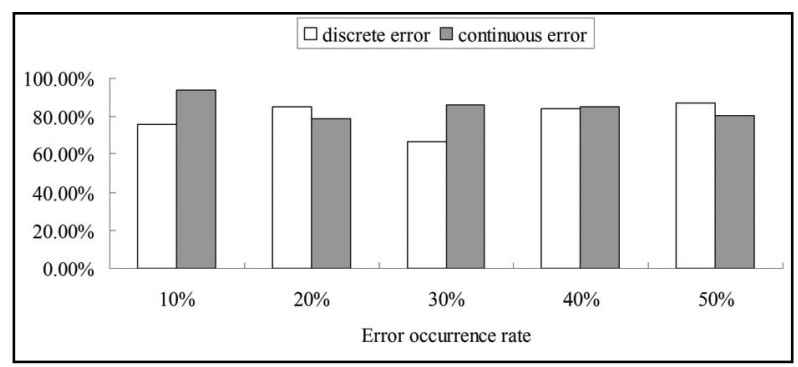

Figure 10. The comparison of distinction ratios.

Table 4. Parameter configuration of the experiment.

\begin{tabular}{|c|c|}
\hline & Parameters \\
\hline $\begin{array}{c}\text { General } \\
\text { parameters }\end{array}$ & $\begin{array}{l}\text { Data amount: } 200 \text { temperature samples per } \\
\text { node with error/event injection; } \\
\text { Error occurrence frequency } \leq 50 \% \text {; } \\
\text { Event occurrence frequency } \leq 1 \% \text {; } \\
\text { Error/Event value range: }[-30,100]\end{array}$ \\
\hline $\begin{array}{c}\text { Step } 1 \\
\text { (node level) }\end{array}$ & $\begin{array}{l}\text { Window length: } 40 \text { samples; } \\
\text { Sliding ratio: } 50 \% \text {. }\end{array}$ \\
\hline $\begin{array}{c}\text { Step } 2 \\
\text { (neighbor level) }\end{array}$ & Average number of 1-hop neighbors: 3 \\
\hline $\begin{array}{c}\text { Step } 3 \\
\text { (cluster level) }\end{array}$ & Average fusion range: 10 nearby nodes \\
\hline $\begin{array}{l}\text { Discrimination } \\
\text { metrics }\end{array}$ & $\begin{array}{l}\text { Correlation coefficient, mean absolute error, } \\
\text { root mean squared error, relative absolute } \\
\text { error and root relative squared error. }\end{array}$ \\
\hline Evaluation metrics & $\begin{array}{l}r_{1} \text {-mistaking errors for ordinary samples; } \\
r_{2} \text {-mistaking errors for events; } \\
r_{3} \text {-mistaking ordinary readings for events; } \\
r_{4} \text {-mistaking ordinary readings for errors; } \\
r_{5} \text {-mistaking events for ordinary samples; } \\
r_{6} \text {-mistaking events for errors; } \\
r_{7} \text {-correct distinction of events, errors, and } \\
\text { ordinary samples; } \\
r_{1}+r_{2}+r_{3}+r_{4}+r_{5}+r_{6}+r_{7}=1 \text {; } \\
\text { False Alarm }=r_{2}+r_{3} \text {; Miss Hit }=r_{5}+r_{6} \text {. }\end{array}$ \\
\hline
\end{tabular}

However, the other metrics are more or less inaccurate over part of the samples. For example, except for the correlation coefficient, all of the other four metrics show fluctuations during time interval $[5063,5154]$ while the raw samples do not change much during this time period. Therefore, correlation coefficient is used to express the value difference between the predicted and the realsensed samples.

The performance of the discrimination framework is evaluated in different cases of error occurrence rate in the network. The ratio $r_{7}$ reflects how many samples are correctly judged in each step over all of the samples. As shown in Figure 12, $r_{7}$ decreases with the increase of error occurrence rate in Steps 1 and 2, but Step 3 has corrected most of the wrong discriminations and kept the ratio as high as $97 \%$ in all of the five cases. Meanwhile, Figure 13 shows that, in different cases of the network, the step-wise processing has always kept an increasing

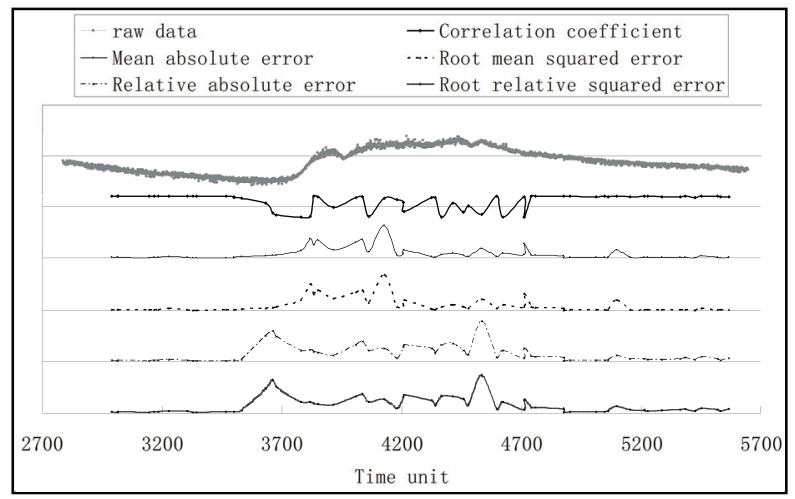

Figure 11. The comparison of different distinction metrics.

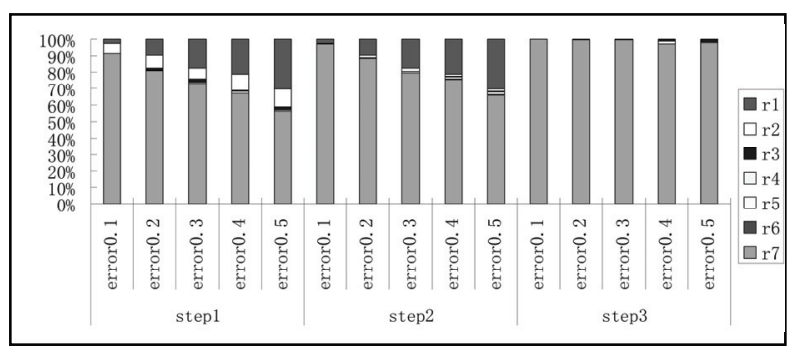

Figure 12. The comparison of different steps in processing.

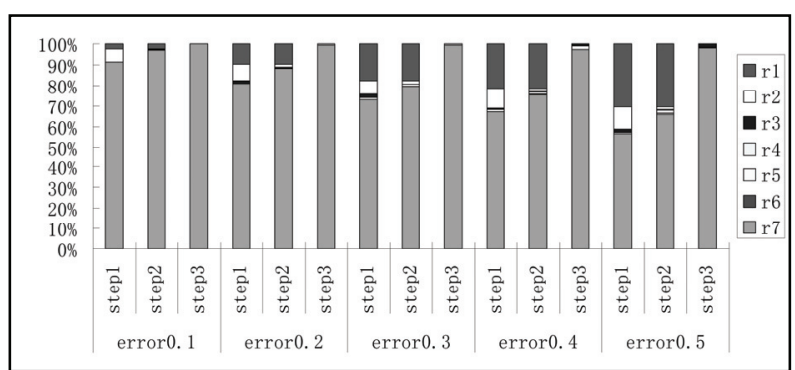

Figure 13. The comparison of different error occurrence frequency. 
trend of the correct distinction (i.e., $r_{7}$ ), demonstrating the robustness of the discrimination framework.

Besides the ratio of correct distinction, the ratios of false alarm and miss hit have been analyzed in Figure 14. Although the false alarm is relatively high in the first step, the processing of the following two steps can significantly reduce the ratio of false alarm. The average false-alarm rates after each step are $9 \%, 0.6 \%$ and $0.5 \%$. On the other hand, the average miss-hit rates after each step are $0.5 \%, 0.8 \%$ and $0.6 \%$. There is slight increase of miss-hit rate after Steps 2 and 3. This is because new errors would be incurred by the cross-comparison and ranking among samples from different nodes. Thus, tradeoffs exist between the false-alarm rate and miss-hit rate in the discrimination framework.

More specifically, there are usually higher false-alarm rates in the solutions to event-detection problems since they mistake erroneous samples for event samples. On the other hand, the approaches of traditional anomaly-detection problems often mistake event samples for erroneous samples and result in higher miss-hit rates. Figure 15 and Figure 16 compare the false-alarm rate

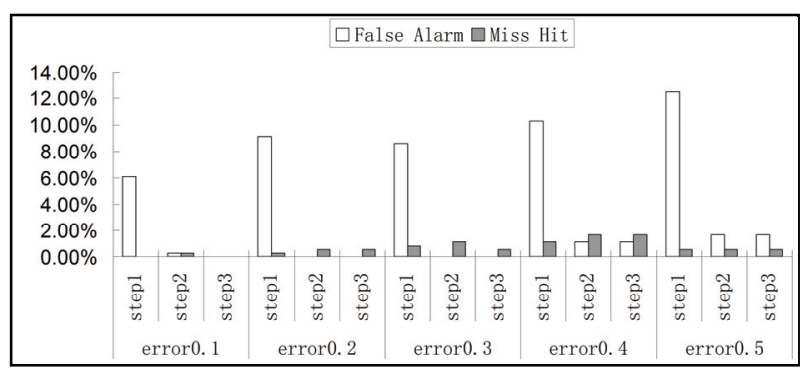

Figure 14. Statistical result of false alarm and miss hit.

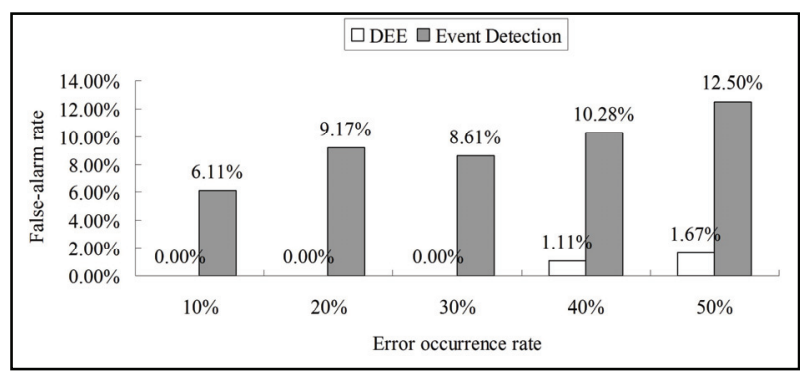

Figure 15. Comparison of false-alarm rate.

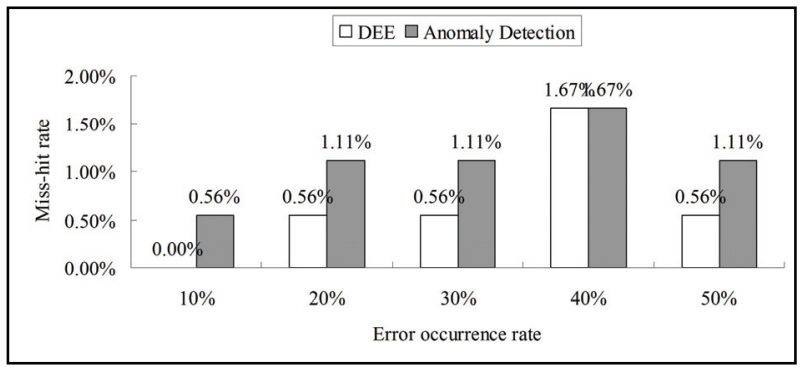

Figure 16. Comparison of miss-hit rate. and miss-hit rate of the discrimination framework for DEE problem with the corresponding methods in event detection and anomaly detection, respectively. It is obvious that the discrimination framework in our work has significantly outperformed the traditional event-detection method in false-alarm rate, and the miss-hit rate of the discrimination framework is much lower than that of the traditional anomaly-detection framework.

\section{Conclusions and Future Work}

Different from the traditional event detection and anomaly detection problems, in this article, we have presented the problem of data discrimination to separate sensor data into the subsets of error samples, event samples and ordinary samples. A multi-level processing framework has been devised in view of the characteristics and constraints of sensor nodes, as well as the requirement of discrimination correctness and response time. Both the scenario-based simulations and the experiment based on real-sensed data have been carried out to evaluate the performance of the discrimination framework. The simulation and experimental results show that different types of monitored events need different kinds of data-discrimination methods and different level of data processing. For example, the correlation coefficient serves as the most appropriate distinction metric in the sample discrimination of durative events. According to the comparison results of false-alarm and miss-hit rate of the methods for traditional event-detection and anomaly-detection problems, the multi-level processing framework has significantly increased the correct distinction ratio, substantially reduced the false-alarm rate, and kept the miss-hit rate in an acceptable low level.

In the future, we plan to apply such a framework to more specific event monitoring problems in fault-prone sensor networks. It is believed that good performance of data discrimination comes from wise use of the domain-knowledge of the event patterns and error patterns as well as the characteristics of wireless sensor networks.

\section{References}

[1] D. Shepherd and S. Kumar, "Microsensor Applications," In: S. S. Iyengar and R. R. Brooks Eds., Distributed Sensor Networks, Chapman \& Hall/CRC Press, 2005, pp. 11-27.

[2] P. Buonadonna, D. Gay, J. M. Hellerstein, W. Hong and S. Madden, "TASK: Sensor Network in a Box," Technical Report, International Research Berkeley, January 2005.

[3] Y. Zhang, M. Nirvana and H. Paul. "Why General Outlier Detection Techniques Do Not Suffice for Wireless Sensor Networks?" Intelligent Techniques for Warehousing and Mining Sensor Network Data, IGI Global, 2009.

[4] B. Sheng, Q. Li, W. Z. Mao and W. Jin. "Outlier Detection in Sensor Networks," Proceedings of the ACM Inter- 
national Symposium on Mobile Ad Hoc Networking and Computing, Montreal, September 2007, pp. 219-228.

[5] T. Palpanas, D. Papadopoulos, V. Kalogeraki and D. Gunopulos, "Distributed Deviation Detection in Sensor Networks," SIGMOD Record, Vol. 32, No. 4, December 2003, pp. 77-82.

[6] S. Subramaniam, T. Palpanas, D. Papadopoulos, V. Kalogeraki and D. Gunopulos, "Online Outlier Detection in Sensor Data Using Non-Parametric Models," Proceedings of Very Large Data Bases, Seoul, 12-15 September 2006, pp. 187-198.

[7] J. W. Branch, C. Giannella, B. Szymanski, R. Wolff and H. Kargupta. "In-network Outlier Detection in Wireless Sensor Networks," Proceedings of 26th International Conference on Distributed Computing Systems, Lisboa, 4-7 July 2006.

[8] M. E. Otey, A. Ghoting and S. Parthasarathy. "Fast Distributed Outlier Detection in Mixed-Attribute Data Sets," International Journal of Data Mining and Knowledge Discovery, Vol. 12, No. 2-3, 2006, pp. 203-228.

[9] D. Janakiram, V. Reddy and A. Kumar, "Outlier Detection in Wireless Sensor Networks Using Bayesian Belief Networks," 1st International Conference on Communica- tion System Software and Middleware, New Delhi, 2006, pp. 1-6.

[10] C. Franke and M. Gertz, "ORDEN: Outlier Region Detection and Exploration in Sensor Networks," Proceedings of ACM Special Interest Group on Management of Data, Providence, 29 June-2 July 2009.

[11] M. Ding, D. C. Chen, K. Xing and X. Z. Cheng. "Localized Fault-Tolerant Event Boundary Detection in Sensor Networks," Proceedings of 24th Annual Joint Conference of the IEEE Computer and Communications Societies, Vol. 2, 13-17 March 2005, pp. 902-913.

[12] V. Chandola, A. Banerjee and V. Kumar, "Anomaly Detection: A Survey," ACM Computing Surveys, September 2009, pp. 1-72.

[13] K. Ni, N. Ramanathan, M. N. H. Chehade, L. Balzano, S. Nair, S. Zahedi, E. Kohler, G. Pottie, M. Hansen and M. Srivastava, "Sensor Network Data Fault Types," ACM Transactions on Sensor Networks, Vol. 5, No. 3, May 2009.

[14] M. M. Kokar, J. A. Tomasik and J. Weyman, "Data vs. Decision Fusion in the Category Theory Framework," Proceedings of Fusion 4th International Conference on Information Fusion, Vol. 1, 2001.

[15] http://db.csail.mit.edu/labdata/labdata.html 